\section{MYELOPATHY IN HEPATIC CIRRHOSIS}

BY

\section{S. S. PANT, M.D.}

A. N. BHARGAVA, F.A.C.A.

M. M. SINGH, M.D.

AND

P. C. DHANDA, M.R.C.P.

From the Department of Medicine, M.A. Medical College, Delhi University, and the Irwin Hospital, New Delhi

Transient neurological changes occurring in patients suffering from hepatic precoma and coma are well known (Adams and Foley, 1953; Summerskill et al., 1956). The development of permanent neurological deficits in such patients has only recently been appreciated. It is becoming increasingly clear that permanent neurological changes referable to many systems are not uncommon in these patients. During the past five years R. D. Adams and M. Victor (personal communication, 1962) have collected 15 cases of cirrhosis with episodes of coma in which permanent intellectual, cerebellar, extrapyramidal, and pyramidal impairment in various combinations occurred.

The occurrence of chronic spastic paraplegia of pure motor type in association with cirrhosis was for the first time clearly documented by Zieve et al. (1960). The two cases reported by these authors had remarkably parallel clinical histories and identical features and course of their myelopathy. Both had portal cirrhosis of the liver. Both had undergone portacaval shunt surgery for relief of symptoms referable to portal hypertension. Both had partial gastrectomy for associated peptic ulcer. Both exhibited protein sensitivity and suffered repeated episodes of protein coma which followed the portacaval shunt surgery. In both patients myelopathy had an insidious onset, was confined solely to the pyramidal tracts of the spinal cord, and was steadily progressive, making the patients bedridden in a matter of a few months. Neither patient had any sensory symptoms or signs and there were no abnormalities attributable to the cranial nerves or to the cerebellar and the extrapyramidal systems. Post-mortem data, available in one of these patients, consisted of the striking pathological finding of marked and selective demyelination in the spinal cord limited to the pyramidal tracts. That the lesion was a primary demyelination was deduced from the fact that the longitudinal sections of the spinal cord demonstrated severe demyelination in the presence of morphologically intact axons. The involvement extended no higher than the cervical cord and became progressively more pronounced caudally. Pyramidal-tract demyelination was not found in the medulla, pons, cerebral peduncles, or internal capsule.

Recently we encountered two cases of chronic spastic paraplegia in association with hepatic cirrhosis and extreme protein intolerance. Appropriate studies failed to incriminate any of the usual causes of myelopathy.

\section{Case 1}

A 48-year-old man was admitted to hospital with the chief complaint of progressive weakness and stiffness of both legs of six months' duration. There was no history of alcoholism, jaundice, haematemesis, or melaena.
Twenty months previously he had been admitted to another hospital because of ascites and oedema of the feet. Splenomegaly and prominent veins over the abdominal wall were noted. The liver was not palpable. Liver biopsy showed portal cirrhosis. With salt restriction, diuretics, and a high-protein diet, ascites and oedema disappeared in a few weeks, but he started having episodes of hepatic precoma characterized by disorientation, confusion, aberrant behaviour, and flapping tremor followed by deep stupor. These episodes lasted a variable period ranging from a few hours to two days. The proteins in the diet were progressively reduced and the episodes stopped only when the intake of proteins was restricted to less than $20 \mathrm{~g}$. a day. Any attempt to increase the protein intake beyond this level was followed by recurrence of episodes of hepatic precoma. After a five-months stay in the other hospital he was discharged on a regimen of salt restriction, weekly diuretics, and severe protein restriction (less than $20 \mathrm{~g}$. a day). He followed this regimen strictly and had no further recurrence of oedema, ascites, or episodes of confusion, disorientation, and stupor.

Six months ago he first became aware of weakness and stiffness in both legs unaccompanied by paraesthesia or pain. Walking became progressively more difficult, and about three months ago he became bedridden.

Physical examination on admission revealed no ascites, oedema, or jaundice. Prominent veins over the abdominal wall were present. Liver was not palpable. A five-finger splenomegaly was noted. He was mentally clear and alert. Abnormal neurological findings were confined to the legs only and consisted of marked bilateral spasticity and weakness, bilaterally hyperactive knee-jerks, bilaterally sustained ankle clonus, and bilaterally extensor plantar responses. Sensory examination, including pain, touch, position, and vibration sense, was entirely normal. There were no abnormalities referable to the cranial nerves or to the cerebellar and extrapyramidal systems.

Investigations revealed normal urinary findings and a nearly normal haemogram, with $\mathrm{Hb} 10 \mathrm{~g} . / 100 \mathrm{ml}$., W.B.C. $6,900 /$ c.mm., and E.S.R. $15 \mathrm{~mm}$. in first hour. No megaloblasts were seen in the bone-marrow smears. Free acid was present in the aspirated gastric juice. The total serum proteins were 6 g. $/ 100 \mathrm{ml}$. (albumin $2.2 \mathrm{~g}$., globulin $3.8 \mathrm{~g}$.). Several liver-function tests gave normal results: serum bilirubin $0.2 \mathrm{mg} . / 100 \mathrm{ml}$., zinc sulphate turbidity 3 units, serum alkaline phosphatase 8 K.A. units, and serum glutamic-oxaloacetic transaminase value of 40 units. A liver biopsy again confirmed the diagnosis of portal cirrhosis. Serum copper and ceruloplasmin values were within normal limits. A slit-lamp examination of the cornea did not show any abnormality. At lumbar puncture, cerebrospinal fluid was under normal pressure and showed normal dynamics. It contained 5 lymphocytes $/$ c.mm., proteins $30 \mathrm{mg}$., chloride $740 \mathrm{mg}$., and sugar $60 \mathrm{mg} . / 100 \mathrm{ml}$. The Wassermann and Kahn tests on both serum and C.S.F. gave negative results. No abnormalities were found in $x$-ray films of cervical, thoracic, and lumbar spine. Myelographic studies showed free flow of dye throughout and there was no evidence of any filling defect anywhere along the spine.

Case 2

A 24-year-old man was admitted to hospital for the second time with the chief complaint of difficulty in walking of about two weeks' duration. One year previously he had been admitted to the surgical ward with the history of three episodes of haematemesis and melaena during the 18 months preceding that admission. Examination at that time revealed a spleen palpable six fingerbreadths below the left costal margin. Liver was not palpable and there was no ascites. Serum bilirubin was $2 \mathrm{mg} . / 100 \mathrm{ml}$. Barium-meal studies showed oesophageal varices. A percutaneous spleno-portal venogram showed dilated splenic and portal veins with marked narrowing of the intrahepatic branches. Liver biopsy showed portal cirrhosis. Nine months ago portacaval anastomosis was performed and at operation portal-vein 
pressure was $300 \mathrm{~mm}$. of water. After a stormy postoperative course, with deepening jaundice requiring intensive use of antibiotics and steroids, he gradually improved, and seven and a half months ago he was discharged from the surgical wards. He has had no haematemesis or melaena since the operation.

About two weeks ago he first became aware of some difficulty in walking and was admitted to the medical wards for study and evaluation. On examination he was mildly jaundiced. The liver was not palpable. A six-finger splenomegaly was present. There was no ascites. Neurologically, there were pyramidal-tract signs in both lower extremities. Both legs were somewhat weak. Their muscle tone appeared normal. Knee-jerks and ankle-jerks were bilaterally hyperactive and plantar responses were bilaterally extensor. Examination of the sensory, cerebellar, and extrapyramidal systems revealed no abnormalities. Cranial nerves were intact and intellectual functions were not blunted.

Serum bilirubin was $4 \mathrm{mg} . / 100 \mathrm{ml}$. A haemogram, including bone-marrow-smear examination, revealed no abnormality. Free acid was present in the gastric juice. C.S.F. findings were normal. Serological tests for syphilis were negative. $X$-ray examination of the spine was normal. Myelographic studies showed free flow of dye. Serum copper and ceruloplasmin values were within normal limits. A slit-lamp examination of the eyes revealed nothing abnormal.

The patient, a strict vegetarian, had a low protein intake, and it was estimated that his intake of proteins did not exceed 20 g. a day. At one stage during his hospital stay his protein intake was increased to $60 \mathrm{~g}$. a day and he promptly went into hepatic precoma on the fourth day. He has been in the ward for over three months and his spastic paraplegia continued to progress.

\section{Discussion}

The two cases reported here had the unusual combination of cirrhosis and chronic spastic paraplegia without any other neurological involvement. Such a combination has previously been reported in only two other patients (Zieve et al., 1960), and the clinical picture, course, and findings in these four patients have many features in common. The underlying liver disease in all of them was cirrhosis. Three of these patients were subjected to portacaval shunt procedure. In the remaining patient the widely dilated veins on the abdominal wall and the high degree of protein intolerance in the absence of any serious derangement of liverfunction tests pointed to the spontaneous development of portacaval shunt. A most striking common denominator in all these four cases was protein sensitivity of a high degree ; none could tolerate more than $20 \mathrm{~g}$. of protein a day. In the three patients subjected to shunt surgery, myelopathic manifestations set in nine months to two years after this procedure. In each case paraplegia was insidious in onset and there was steady progression. Three of the four patients became bedridden in six to nine months after onset, and in the remaining patient, with a total duration of neurological disease of only three and a half months, a similar course is emerging. None of these patients showed any abnormality referable to the cranial nerves or to the sensory, cerebellar, and extrapyramidal systems.

Permanent neurological abnormalities in association with cirrhosis of the liver are found in Wilson's disease. There are, however, no reports of isolated pyramidaltract involvement in Wilson's disease. In the two cases reported here this diagnosis can be easily dismissed because of the late onset of neurological manifestations, the normal serum copper and ceruloplasmin levels, the absence of Kayser-Fleischer rings, and no evidence of extrapyramidal involvement.

The possibility that the hepatic cirrhosis and the myelopathy in these cases were merely chance associations must also be considered. The clinical course, the normal $x$-ray films of the entire spine, the normal myelographic appearances, the normal bone-marrow findings, the presence of free acid in the gastric juice, and the negative serological tests for syphilis rule out the common causes of chronic spastic paraplegia. However, many cases of chronic spastic paraplegia with isolated pyramidal-tract involvement do not yield themselves to any specific diagnosis (R. D. Adams, personal communication, 1961), and the question, therefore, must remain whether these patients had some idiopathic type of motor myelopathy unrelated to the liver disease.

A causal relationship between the liver and cord disease is suggested by the pathological data available in only one case so far (Zieve et al., 1960). There was massive and selective primary demyelination of the pyramidal tracts in the cord. Brown (1957) examined the spinal cord in five cases of fatal hepatic coma and mentioned that two cases showed "demyelination of a mild degree in the lateral columns" though no correlated clinical symptoms had been noted. It would therefore seem that minor degrees of demyelination of no clinical significance are not uncommon in the lateral columns of the cord in fatal hepatic coma. The severe demyelination of clinical significance seen in Zieve's necropsied case could therefore be interpreted as representing an advanced degree of the same cord pathology as is commonly seen in severe liver disease. A final answer must await the reporting of further cases of liver disease with myelopathy and further study of cord pathology in liver disease.

\section{Summary}

Two cases of purely motor myelopathy with spastic paraplegia in association with hepatic cirrhosis are reported.

Both patients had predominant portacaval shunt mechanism, surgically acquired in one case and spontaneously developing in the other.

Both patients showed a severe degree of protein intolerance.

The motor myelopathy was characterized by insidious onset and steady progression leading to incapacitation in a few months. There was complete absence of neurological findings except those referable to pyramidal tracts in the cord.

Two similar cases so far reported in the literature are reviewed and the significance of this association of myelopathy with liver disease is briefly discussed.

\section{REFERENCES}

Adams, R. D., and Foley, J. M. (1953). Ass. Res. nerv. Dis. Proc., 32, 198

Brown, I. A.' (1957). Liver-Brain Relationship. Thomas, Springfield, Illinois.

Summerskill, W. H. J., Davidson, E. A., Sherlock, S., and Steiner, R. E. (1956). Quart. J. Med., 25, 245.

Zieve, L., Mendelson, D. F., and Goepfert, M. (1960). Ann. intern. Med., 53, 53.

The Secretary of State for Scotland has confirmed an Order made by the Town Council setting up another smoke control area in Clydebank. The area comprises some 62 acres and affects 1,516 houses. The Order, the Clydebank Central Redevelopment Smoke Control Area Order, 1963, will come into force on October 1 . 